\title{
Youssef's Syndrome - a complication of corrective surgery for mullerian anomaly
}

\author{
Vineet Mishra $^{1}$, Suwa Saini ${ }^{2}$, Rohina Aggarwal ${ }^{1}$, Priyankur Roy $^{1}$, Shaheen Hokabaj ${ }^{1}$
}

\begin{abstract}
${ }^{1}$ Department of Obstetrics and Gynaecology, Institute of Kidney Disease and Research Centre, Ahmadabad, Gujarat, India

${ }^{2}$ Department of Obstetrics and Gynaecology, SP Medical College, Bikaner, Rajasthan, India
\end{abstract}

Received: 06 September 2016

Accepted: 03 October 2016

\author{
*Correspondence: \\ Dr. Vineet Mishra, \\ E-mail: vineet.mishra.ikdrc@gmail.com
}

Copyright: $\odot$ the author(s), publisher and licensee Medip Academy. This is an open-access article distributed under the terms of the Creative Commons Attribution Non-Commercial License, which permits unrestricted non-commercial use, distribution, and reproduction in any medium, provided the original work is properly cited.

\begin{abstract}
The presence of cervicovaginal agenesis with unicornuate uterus is a very rare mullerian anomaly. Presence of functioning unicornuate uterus poses as a great challenge for gynecologist, because a successful repair could restore normal menses and may preserve the patient's fertility. Though, a complication of this surgery (utero-vaginal anastomosis) is Youssef's Syndrome. We, hereby report a case of 16 year old unmarried female with complete cervicovaginal agenesis with unicornuate uterus, who initially underwent vaginoplasty (McIndoe's) along with uterovaginal anastomosis in order to preserve her uterus, but subsequently required hysterectomy due to Youssef's Syndrome.
\end{abstract}

Keywords: Cervicovaginal agenesis, Hysterectomy, Vaginoplasty, Youssef's Syndrome

\section{INTRODUCTION}

Mullerian duct anomalies (MDA) are congenital defects of the female genital system that arise from abnormal embryological development of the Mullerian ducts. ${ }^{1}$ These abnormalities can include failure of development, fusion, canalization, or reabsorption, which normally occurs between 6 and 22 weeks in utero. ${ }^{2}$ Cervicovaginal agenesis in presence of uterus is very rare condition. ${ }^{3}$ The prevalence of vaginal agenesis is 1 in 5000 live female birth, whereas that of cervical agenesis is 1 in 100000 live birth. ${ }^{4}$ These patients are phenotypically female with normal female genotype and normal endocrine status. The successful surgical approach till date for this condition is neovagina creation, followed by transabdominal approach to create a communication between uterus and vagina by application of stents (which are usually removed after healing of the tract). Unfortunately, every surgery has its complications and one of the known dreaded complications of this surgery is the Youssef's Syndrome.
This syndrome was firstly described in literature in 1938, however the term was coined by Yossef AM. ${ }^{6}$ Vesicouterine fistula is a communication between bladder and the uterus. It is one of the urogenital fistulas which are least common in occurrence with an incidence of only about 1-4 \%. ${ }^{6}$ Its main symptoms are cyclic haematuria (menouria), amenorrhoea, urinary incontinence and recurrent UTI. The triad of menouria, amenorrhea and urinary continence is known as Youssef's Syndrome.

\section{CASE REPORT}

A 16 year old unmarried girl, presented to our Gynaecology OPD in December, 2015 with chief complaints of primary amenorrhea with cyclical lower abdominal pain, generalized weakness and facial puffiness. She was a known case of nephrotic syndrome since 2 years, for which she was on oral steroids on and off. She was also a known case of epilepsy and was on Tab. Leviteracetam for the same. There was no past history of any surgery. On examination, her vital signs were stable. Her skeletal, cardiovascular and respiratory 
system examinations were normal. Her secondary sexual characteristics were appropriate for age and Tanner stage for breast and pubic hair was stage IV. On abdominal examination, no mass was palpable but deep tenderness was present. Pelvic examination revealed vaginal dimple without any vaginal orifice. On rectal examination, no mass was palpable. She was admitted with plans of evaluation followed by corrective surgery, if required.

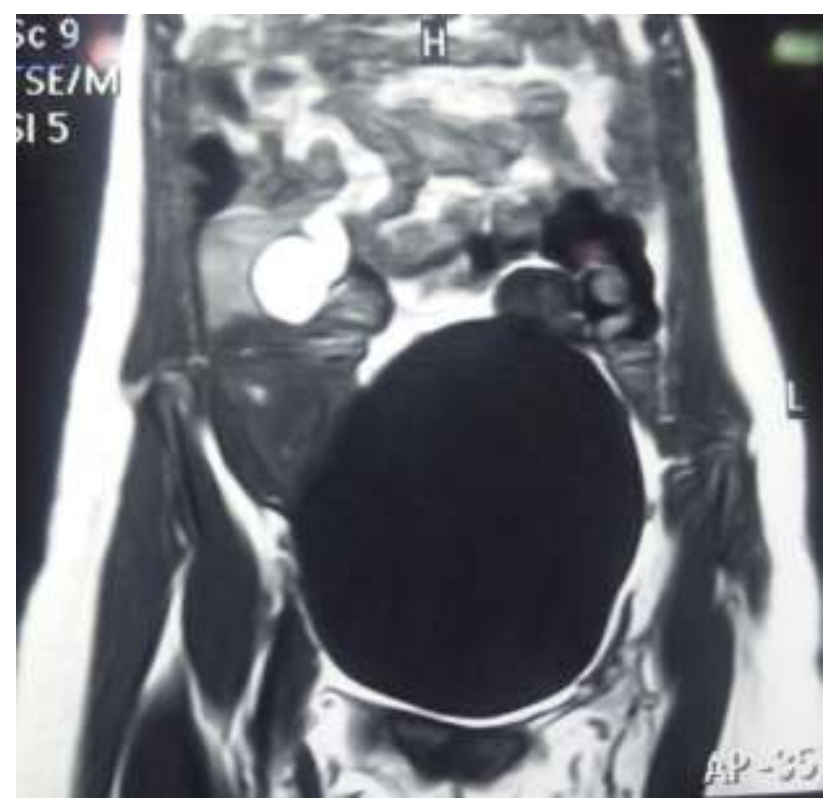

Figure 1: Ultrasonography and MRI scanning.

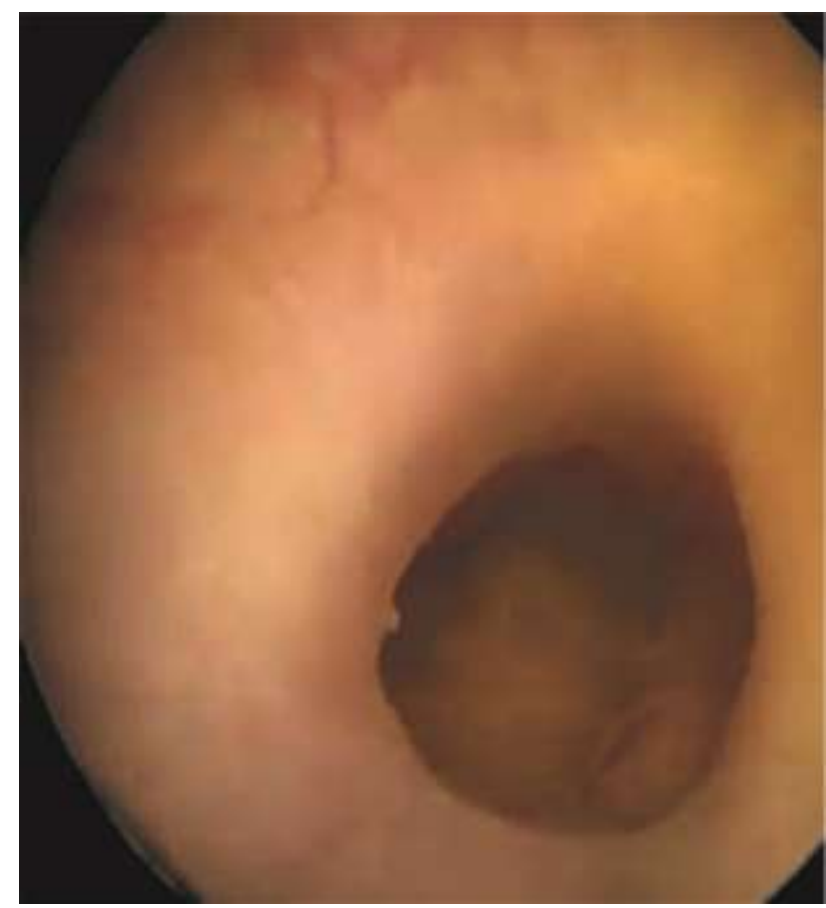

Figure 2: Diagnosing with Youssef's Syndrome.

On admission, her blood tests except for serum creatinine levels $(1.58 \mathrm{mg} / \mathrm{dl})$ were found to be normal. Her urine routine microscopy examination revealed presence of proteinuria $(>500 \mathrm{md} / \mathrm{dl})$. Her abdomino-pelvic ultrasonography revealed 4.1 x $2.1 \mathrm{~cm}$ endometrial collection suggestive of haematometra, along with presence of $4.6 \times 4.3 \mathrm{~cm}$ complex cystic lesion in right adnexa suggestive of haematosalpinx. Her left adnexa were normal. Her left kidney was small $(3.1 \mathrm{x} 1.6 \mathrm{~cm}$ size) with loss of cortico-medullary differentiation. Her MRI scanning also revealed similar findings with presence of haematometra and right haematoalpinx with blind ended uterus, and absent cervix and vagina. Her left kidney was small. Both her ovaries were normal and were at the level of pelvic brim. Her right kidney was normal. Her karyotyping was normal with 46 (XX) chromosomes.

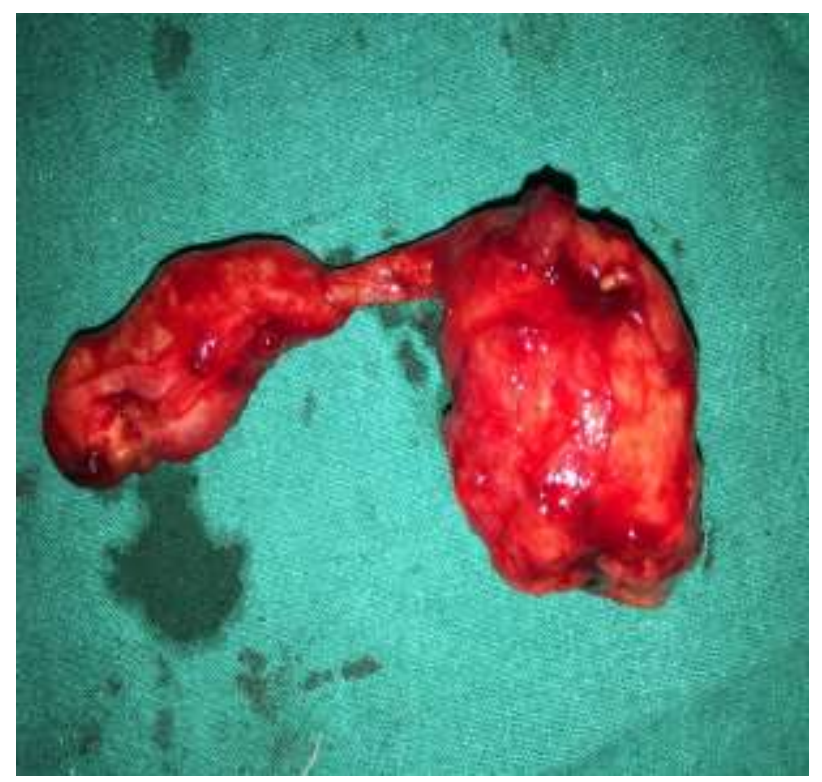

Figure 3: Abdominal hysterectomy with unilateral salpingectomy.

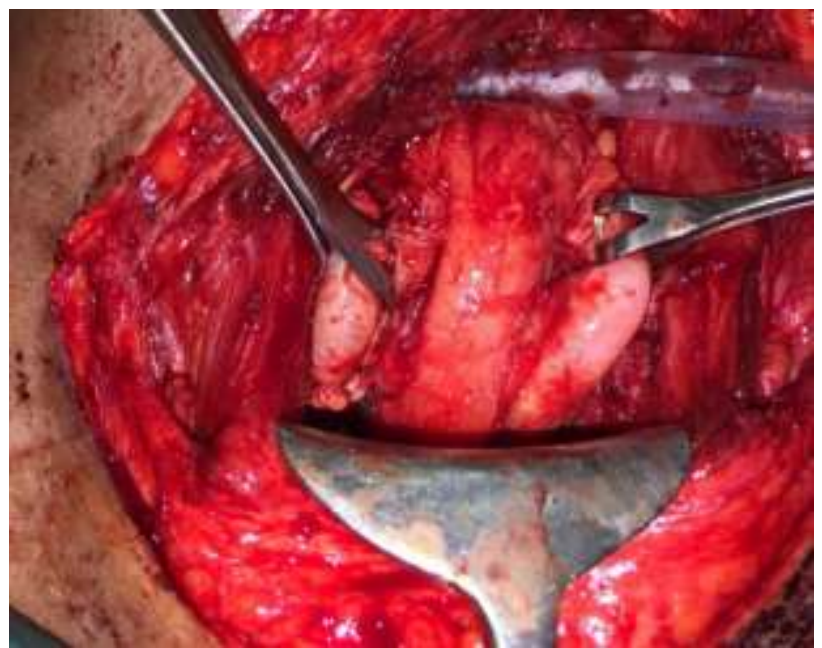

Figure 4: Normal bilateral ovaries.

After proper counselling of the patient's relatives, she was planned for creation of neovagina and uterovaginal anastomosis. Neovagina was created by McIndoe's method using partial thickness skin graft, taken from left 
thigh. On laparotomy, there was right side unicornuate uterus with right sided haematosalpinx, and left side rudimentary horn with normal left fallopian tube. Bilateral ovaries were normal. A stab incision was given on the uterus and hemorrhagic collection was drained. Uterovaginal anastomosis was performed and malecot's catheter was kept, such that it served as a communication between uterus and the vagina

On $8^{\text {th }}$ postoperative day, vaginal mold was removed and diagnostic vaginoscopy was done. Skin graft was healthy. A new mold was placed in the vagina. The patient and her mother were educated about daily change and care of vaginal mold.

She had normal menstrual cycles for 2 months. After her second cycle she reported to our Gynaecology OPD with complaints of passing out the malecot's catheter. She was advised to follow-up after her next menstrual cycle with the hope that the utero-neovaginal anastomosis would be patent. She came for follow-up again after 2 months with complaints of amenorrhea. Ultrasonography and MRI scanning was performed and she was detected to have a large hematometra of size $5.7 \times 5.3 \times 4.7 \mathrm{~cm}$ (Figure 1) and also unilateral hematosalpinx. Thus, a repeat surgery was planned for her.

She underwent utero-vaginal reanastomosis. On laparotomy, right sided unicornuate uterus with right haematosalpinx was noted. A stab incision was given on the uterus and hemorrhagic collection was drained. Hysteroscope was introduced through the same stab incision. A nick was given from below vaginally, under the guidance of hysteroscopic light. After making the nick, the scope was removed and Kocher's forceps was introduced through the uterine incision. Malecot's catheter was inserted by rail road method and sutured to the anterior wall of the uterus. On post-operative day 3, after removing the foley's catheter, she started leaking urine per-vaginum. With a clinical diagnosis of urinary fistula, she was recatheterized and discharged with advice to follow-up after 15 days.

On her $25^{\text {th }}$ post-operative day, she came to Gynae OPD with complaints of hematuria which lasted for 3 days. She also gave history of falling down of the malecot's catheter (stent) two weeks after discharge from the hospital. She was readmitted and vaginoscopy followed by cystoscopy was performed. Vaginoscopy revealed blind vagina. Cystoscopy showed a small rent in the posterior wall of the bladder. She was thus, diagnosed to have Youssef's Syndrome (Figure 2). She was discharged with foley's catheter insitu and advised to come back after 6 weeks for definitive surgery.

After 6 weeks she was readmitted and Abdominal Hysterectomy with unilateral Salpingectomy (Picture - 3) was performed on her. Her bilateral ovaries, being normal were preserved (Picture - 4). The fistulous tract on the posterior wall of the urinary bladder was identified on cystoscopy and subsequently repaired on laparotomy. Omentum patch was placed on the repaired site of the bladder. She was discharged on post-operative day 7 after suture removal with adviced to come back after 21 days for foley's catheter removal.

She is now on regular follow-up and does not have any recurrent symptoms.

\section{DISCUSSION}

Normal vagina develops from the fusion of mesodermal mullerian duct and the endodermal urogenital sinus. Upper $2 / 3^{\text {rd }}$ of the vagina is formed by mullerian tubercle and lower $1 / 3^{\text {rd }}$ by urogenital sinus. Mullerian agenesis can be partial or complete. Partial mullerian agenesis is rare, characterized by normal uterus and small vaginal pouch distal to the cervix, whereas complete mullerian agenesis (MRKH Syndrome) is most common variant encountered. $^{7}$ Vaginal agenesis is most commonly associated with MRKH syndrome. ${ }^{8}$ It is characterized with normal female genotype, phenotype and normal endocrine status. These patients may have a rudimentary horn or total absence of uterus. Their ovaries are normal and the secondary sexual characteristics develop normally.

Diagnosis is usually done by typical history of primary amenorrhea with cyclic lower abdominal pain. Clinical examination usually reveals a blind vagina. Transabdominal or trans-perineal ultrasonography may specify the level of obstacle and agenesis but are not very reliable for the diagnosis. MRI appears to be most reliable investigation for the diagnosis of utero-vaginal malformation and other associated malformations. ${ }^{9}$ IVP and karyotyping should be performed to rule out other malformations and come to the diagnosis.

Previously the recommended treatment of cervicovaginal agenesis was hysterectomy because of high failure rate of canalization procedures and risk of serious ascending infection. But, this approach has been replaced by conservative approach due to recent advances in minimal surgery. ${ }^{10}$ Basic surgical principle is to create neovagina. However, even after surgery, patients have a long term risk of stenosis, necessitating further surgery. Repeated episodes of hematometra, endometriosis and recurrent obstruction may require hysterectomy as a last option. $^{11}$

Youssef's syndrome many a times is iatrogenic, most commonly due to obstetric operations but can also happen due to surgeries for utero-vaginal anastomosis. It is characterised by cyclic hematuria, amenorrhea, and urinary tract infection. Patients usually don't have complaint of urinary incontinence, but urinary incontinence can occur if the level of the VUF is at or below the level of internal os or if the os is incompetent. Most of these cases have delayed presentation ranging 
from few weeks to years following the causative procedure.

The fistula is often diagnosed by imaging studies and diagnostic cystoscopy. On ultrasonography, the fistulous tract is seen as double echogenic lines between anterior uterine wall and posterior bladder wall. ${ }^{12}$ TVS (transvaginal sonography) can routinely be used as a diagnostic modality for VUF. ${ }^{12}$ MRI is the investigation of first choice in diagnosing VUF. ${ }^{13}$ Treatment of VUF includes expectant, medical and surgical management. Expectant management, i.e., wait and watch has also proven successful as in some cases; it can cause spontaneous closure of fistula. Medical management involves induction and maintenance of prolonged amenorrhea with Oral contraceptive pills, progestational agents and gonadotropin releasing hormone analogs. Surgery is however the definitive treatment of choice.

Surgical treatment usually consists of hysterectomy followed by closure of bladder fistula opening. This radical approach has been replaced by conservative uterus preserving approach, and repair of fistulous tract sometimes, but the patient usually lands up with hysterectomy at a later date. Increased morbidity, long hospital stay, and increased blood loss are usually the drawbacks of the surgery, which can be overcome by the laparoscopic approach. ${ }^{12}$ Though, previous laparotomies make the laparoscopic approach difficult due to dense adhesions.

\section{CONCLUSION}

Cervicovaginal agenesis in presence of uterus is a rare condition. Preservation of the uterus should always be attempted in such patients. Though, vesico-uterine fistula (Youssef's Syndrome) is an iatrogenic complication of the same surgical attempt. It is a rare condition, but its incidence has risen of late. It is due to an increase in awareness in individuals about menstrual problems, good imaging modalities, early diagnosis of mullerian anomalies and the advances in performing genital tract corrective surgeries. However by being vigilant, one can try to prevent, diagnose and manage it early, before it is too late for creating socioeconomic and psychological burden to the patient and her family.

Funding: No funding sources

Conflict of interest: None declared

Ethical approval: Not required

\section{REFERENCES}

1. Heinonen PK. Uterus didelphys: a report of 26 cases. European Journal of Obstetrics and Gynecology and Reproductive Biology. 1984;17(5):345-50.

2. Heinonen PK. Clinical implications of the didelphic uterus: long-term follow-up of 49 cases. European Journal of Obstetrics and Gynecology and Reproductive Biology. 2000;91(2):183-90.

3. Abali R, Kuvat SV, Bozkurt S, Kayhan A, Yuksel MA, Caliskan H. Report of surgical correction of a cervicovaginal agenesis case: cervicovaginal reconstruction with pudendal thigh flaps. Archives of Medical Science. 2013;9(1):184-7.

4. Saxena, AK, Herman MI. Vaginal atresia. Webpage. Cited 02 June, 2009.

5. Creighton SM, Daves MC, Cutner A. Laparoscopic management of cervical agenesis. Fertil Steril. 2006;85:1510-3.

6. Youssef AF. Menouria following lower segment cesarean section: a syndrome. American Journal of Obstetrics and Gynecology. 1957;73(4):759-67.

7. Jones HW, Wheeless CR. Salvage of the reproductive potential of women with anomalous development of the mullerian ducts. American Journal of Obstetrics and Gynaecology. 1969;104:348-52.

8. Saraf S, Saraf P. McIndoe vaginoplasty: revisited. International Journal of Gynaecology and Obstetrics. 2007:6.

9. Vallerie AM, Breech L. Update in Mullerian anomalies: diagnosis, management and outcomes. Current Opinion in Obstetrics and Gynecology. 2010;22(5):381-3.

10. Deffanges JV, Haddad B, Muset R, Paniel BJ. Uterovaginal anastomosis in women with uterine cervix atresia - long term follow up and reproductive performance: A study of 18 cases. Hum Reprod. 2001;16:1722-5.

11. McIndoe A. The treatment of congenital absence and obliterative conditions of vagina. Br J Plast Surg. 1950;2(4):254-67.

12. Perveen K, Gupta R, Al-Badr A, Hemal AK. Robot assisted laparoscopic repair of rare post-cesarean section vesicocervical and vesicouterine fistula: a case series of a novel technique. Urology. 2012;80(2):477-82.

13. Narayanan P, Nobbenhuis M, Reynolds KM, Sahdev A, Reznek RH, Rockall AG. Fistulas in malignant gynaecologic disease: etiology, imaging, and management. Radiographics. 2009;29(4):1073-83.

Cite this article as: Mishra V, Saini S, Aggarwal R, Roy P, Hokabaj S. Youssef's Syndrome - a complication of corrective surgery for mullerian anomaly. Int J Reprod Contracept Obstet Gynecol 2016;5:4091-4. 